First publ. in: Physical Review B 68 (2003), 134426

PHYSICAL REVIEW B 68, 134426 (2003)

\title{
Direct observation of spin configuration and classificatio of switching processes in mesoscopic ferromagnetic rings
}

\author{
M. Kläui, ${ }^{*}$ C. A. F. Vaz, and J. A. C. Bland ${ }^{\dagger}$ \\ Cavendish Laboratory, University of Cambridge, Madingley Road, Cambridge, CB3 OHE, United Kingdom \\ T. L. Monchesky and J. Unguris \\ National Institute of Standards and Technology, Gaithersburg, Maryland 20899, USA \\ E. Bauer \\ Department of Physics and Astronomy, Arizona State University, Tempe, Arizona 85287-1404, USA \\ S. Cherifi S. Heun, and A. Locatelli \\ Sincrotrone Trieste, 34012 Basovizza, Trieste, Italy \\ L. J. Heyderman \\ Laboratory for Micro- and Nanotechnology, Paul Scherrer Institut, CH-5232 Villigen PSI, Switzerland \\ Z. Cui \\ Rutherford Appleton Laboratory, Chilton Didcot OX11 0QX, United Kingdom
}

\begin{abstract}
Using nonintrusive imaging techniques we have directly observed the nanoscopic details of the magnetization configuration of epitaxial and polycrystalline mesoscopic ring structures with a $<15-\mathrm{nm}$ resolution. We have found head to head domain walls with different spin structures depending on ring width. Further, we can classify the geometry dependent switching processes according to the number of transitions (single, double, triple) that a ring undergoes in a hysteresis cycle. In the case of triple switching we fin a state with a complete vortex core present in the ring.
\end{abstract}

Understanding and controlling the magnetic properties of small ferromagnetic elements is a major challenge in the rapidly evolving fiel of nanoscale science. This is not only due to the fact that these structures allow for the investigation of fundamental physical properties ${ }^{1}$ but they also have important applications such as in magnetic random access memory cells. ${ }^{2}$ One key issue is to understand and control the magnetic switching precisely. To achieve this one needs, first to have well define and reproducible remanent states and, second, the switching process itself must be simple and reproducible. A possible geometry that fulfill these criteria is the ring geometry, and in particular narrow ferromagnetic rings, which have recently become the focus of intense interest. ${ }^{3-8}$ In all the ring geometries investigated so far, macroscopic measurements and micromagnetic simulations suggest the existence of two magnetic states: ${ }^{3,4,6,7}$ the flux-closur vortex state and the "onion" state, accessible reversibly from saturation and characterized by the presence of two opposite head to head walls. However, since it is the microscopic spin configuratio of the magnetic states that governs the macroscopic magnetic properties and switching, nonintrusive imaging of the microscopic details of the magnetization including the exact spin structure of the head to head domain walls at the nanometer scale is needed in order to understand and control the switching processes. ${ }^{3,9-11}$ Furthermore the geometry and the magnetocrystalline anisotropy are predicted to determine the microscopic spin structure of the magnetic states, ${ }^{9,12}$ for which direct experimental evidence so far is unavailable.
In this paper we use non-intrusive imaging techniques based on photoemission electron microscopy (PEEM), and for higher resolution, scanning electron microscopy with polarization analysis (SEMPA) to directly observe the different magnetization configuration present in polycrystalline and epitaxial ring geometries, including details of the different types of head to head domain walls. Furthermore, the geometry and material dependence of the switching is comprehensively studied using both the magneto-optical Kerr effect (MOKE) and micromagnetic simulations. We fin additional states and transitions that allow us to classify the observed switching behavior of all mesoscopic rings into three categories. Two methods were used to fabricate micrometer-scale rings, lift-off of polycrystalline $\mathrm{Co}$ and Permalloy (NiFe) film grown on naturally oxidized $\mathrm{Si}(001)$ (Ref. 5) and epitaxial growth of $\mathrm{Co}$ on prepatterned $\mathrm{Si}(001)-\mathrm{H}$ substrates. ${ }^{3}$ For the latter method, the epitaxial rings were obtained by depositing a trilayer $\mathrm{Cu}(100) / \mathrm{fcc} \mathrm{Co}(100) / \mathrm{Cu}(100)$ capped with 4-nm Au on a $\mathrm{Si}(100)$ prepatterned wafer in a ultrahigh vacuum molecular beam epitaxy (UHV MBE) system (base pressure $3 \times 10^{-10}$ mbar)..$^{3}$ The lift-off rings were fabricated by means of a PMMA mask that was patterned by e-beam writing. ${ }^{13}$ This was followed by MBE deposition in UHV and a lift-off of polycrystalline Co or Permalloy layers and 4 $\mathrm{nm}$ of $\mathrm{Au}$ to prevent oxidation.

To image the magnetization configuration we chose PEEM and SEMPA, which are nonintrusive techniques in contrast to imaging techniques such as magnetic force microscopy (as shown in MFM imaging of octagonal struc- 

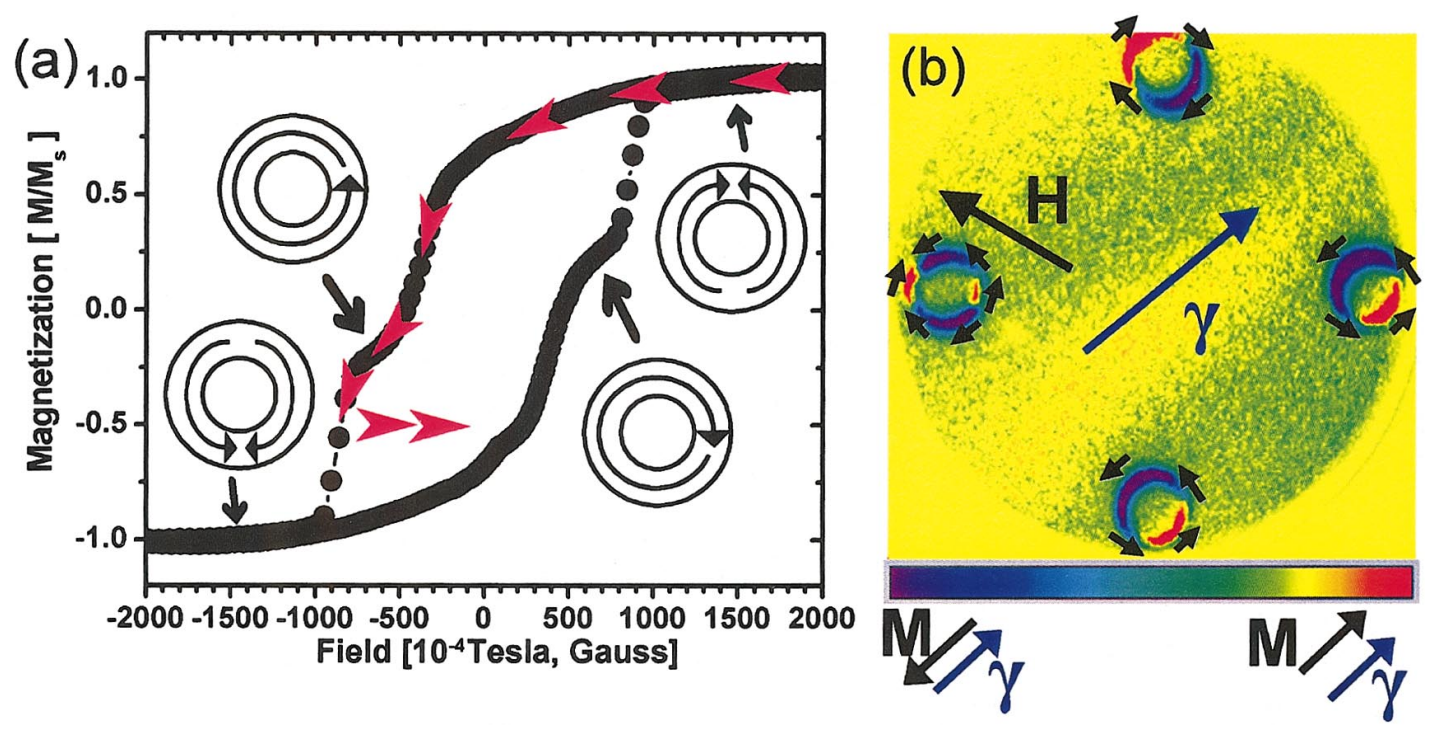

FIG. 1. (Color) (a) Hysteresis loop measured on an array of rings (outer diameter $D=1200 \mathrm{~nm}$, inner diameter $d=900 \mathrm{~nm}$, and thickness $t=15 \mathrm{~nm}$ polycrystalline Co). The magnetization configuration of the onion and the vortex states are shown schematically. Red arrows indicate the fiel path used to obtain the rings in the states imaged with PEEM in (b). (b) PEEM image of four polycrystalline Co rings. The top ring is in the clockwise vortex state, the bottom and right rings are in the counter-clockwise vortex state, and the left ring is in the onion state pointing along the direction of the applied fiel $H$. The fiel of view is $\approx 10 \mu \mathrm{m}$ across and the blue arrow points in the direction of the photon beam. The color scale indicates the direction of the magnetization with reference to the direction of the incoming photon beam [magnetization parallel to the incoming photon beam (red) to antiparallel (purple)].

tures, MFM can influenc and even switch the magnetic configurations ${ }^{6}$ ) Furthermore, unlike MFM, the nonintrusive techniques used here allow us to image even magnetic states with no stray fiel such as the vortex state. PEEM was carried out at the synchrotron ELETTRA in Trieste. ${ }^{14}$ In x-ray magnetic circular dichroism PEEM (XMCDPEEM) the yield of secondary electrons created by excitation with circularly polarized light depends on the dot product $\mathbf{P} \cdot \mathbf{M}$ of the polar- ization $\mathbf{P}$ and the magnetization direction $\mathbf{M}$. The secondary electrons are used for imaging by a SPELEEM (Spectroscopic Photo Emission and Low Energy Electron Microscope) and the yield difference is visible as magnetic contrast in the images. ${ }^{14}$ To achieve a higher resolution than that possible with MFM or PEEM, scanning electron microscopy with polarization analysis (SEMPA) was performed at the National Institute of Standards and Technology (NIST). ${ }^{15}$ In
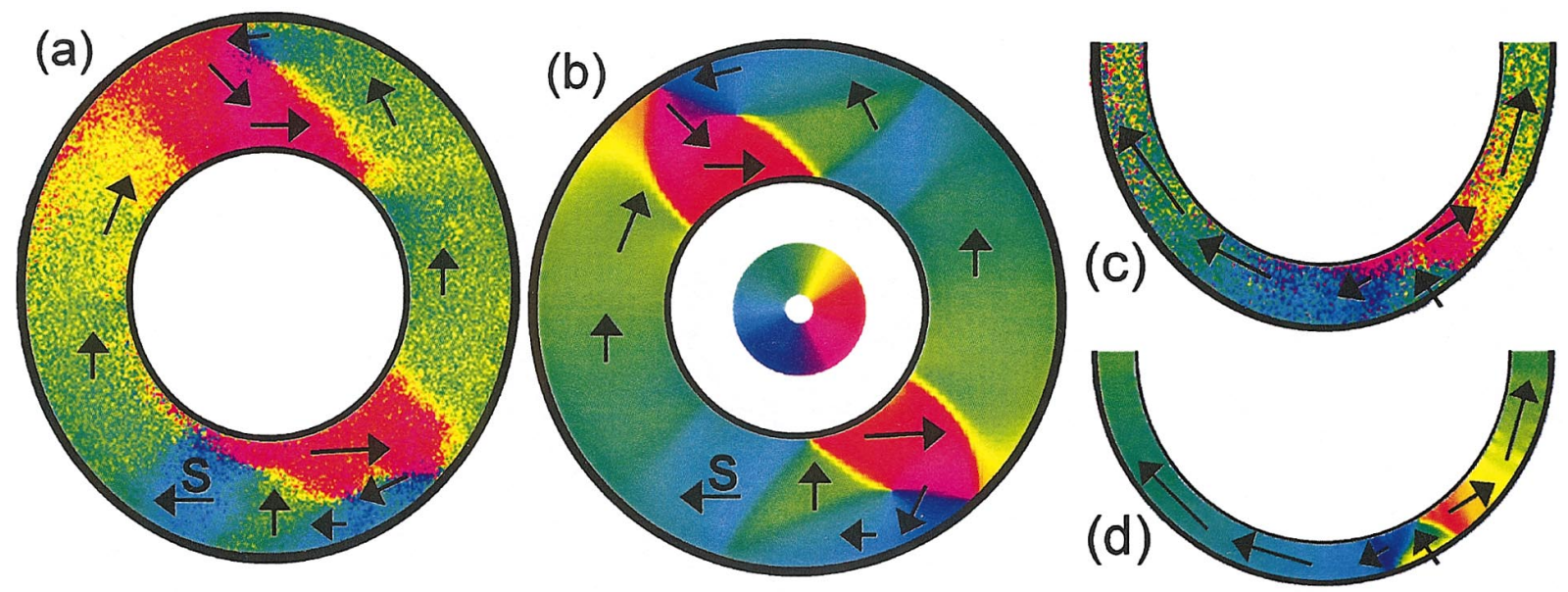

FIG. 2. (Color) Micromagnetic calculations and SEMPA images of the onion state in a wide $(D=1700 \mathrm{~nm}, d=900 \mathrm{~nm})$ and a narrow $(D=1700 \mathrm{~nm}, d=1200 \mathrm{~nm}$ ) epitaxial 34-nm fcc Co ring. (a) SEMPA image of the wide ring. The vortex walls and the magnetocrystalline anisotropy induced domain structure (marked with $S$ ) is visible. (b) Micromagnetic simulation of the wide ring. In addition to the vortex head to head domain wall, the domain structure is visible (marked with $S$ ). The position of the vortex core is slightly different from the SEMPA graph as in the experiment the position is determined by non-magnetic defects that pin the vortex core, which are absent in the simulation. The color code indicating the direction of the magnetization is shown. (c) SEMPA image of the narrow ring (only half the ring is shown). No domain structure is seen as the local shape anisotropy dominates over the magnetocrystalline anisotropy. A transverse head to head domain wall is present. (d) Micromagnetic simulation of the narrow ring exhibiting the transverse domain wall. 
this technique, a scanning electron microscope geometry is extended to include spin polarization sensitive detection of the scattered electrons. In this study SEMPA was used to measure the in-plane magnetization direction. To investigate the switching behavior, MOKE was used to measure hysteresis loops as described in Ref. 3. Simulations were computed by solving the micromagnetic equilibrium equation for each applied fiel on a square mesh, with a 4-nm cell size. A conjugate gradient solver and the OOMMF package ${ }^{16}$ were used for the energy minimization and yielded similar results. The intrinsic parameters used for Co are $M_{s}=1424$ $\times 10^{3} \mathrm{~A} / \mathrm{m}, A=33 \times 10^{-12} \mathrm{~J} / \mathrm{m}$, and $K_{1}=6.5 \times 10^{4} \mathrm{~J} / \mathrm{m}^{3}$ for epitaxial rings, while $K_{1}=0$ is used in the polycrystalline case. For Permalloy we used $M_{s}=800 \times 10^{3} \mathrm{~A} / \mathrm{m}$ and $A$ $=13 \times 10^{-12} \mathrm{~J} / \mathrm{m}$. As shown in Ref. 3, we can use an appropriate minor loop fiel path to obtain rings in the onion state or in the vortex state at remanence. Since we measure an array of rings with a switching fiel distribution we can switch some of the rings into the onion state while some will remain in the vortex state by following the fiel path indicated by the red arrows in the hysteresis loop in Fig. 1(a), where the fiel is relaxed to zero from the middle of the switching fiel distribution of the vortex to the onion transition. ${ }^{3}$ A low resolution PEEM image of four rings (outer diameter $D=1100 \mathrm{~nm}$, inner diameter $d=850 \mathrm{~nm}$ and thickness $t=15 \mathrm{~nm}$ polycrystalline $\mathrm{Co}$, taken at remanence, is shown in Fig. 1(b). As expected some rings are still in the vortex state (two rings have a counter-clockwise and one ring has a clockwise circulation direction) while one ring has already switched into the onion state. While it is easy thus to confir the vortex and onion states with PEEM, the maximum resolution achieved $(30 \mathrm{~nm})$ is not sufficien to resolve the details of the exact magnetization configuratio such as the structure of the head to head domain walls in the onion state.

To obtain more detailed, higher resolution images we use SEMPA. A higher resolution is essential since micromagnetic simulations predict different types of head to head domain walls. ${ }^{8}$ It is the type of domain wall that characterizes the onion state and is very important to for the switching behavior investigated in Ref. 9 and for the nucleation-free onion to vortex transition. ${ }^{3}$ For epitaxial samples (e.g., fcc cobalt), micromagnetic simulations predict that, in addition to the head to head domain walls that are also present in the onion state of polycrystalline rings, epitaxial samples should show additional domainlike features due to the cubic anisotropy. Furthermore, epitaxial samples of different geometries also allow the study of the interplay between the magnetocrystalline anisotropy and the local shape anisotropy that is due to the dipolar interactions in this particular geometry. A SEMPA image for a wide epitaxial ring $(D=1600 \mathrm{~nm}, d=900 \mathrm{~nm}$, and $t=34 \mathrm{~nm} \mathrm{fcc} \mathrm{Co}$ ) is shown in Fig. 2(a). In this wide ring the head to head wall is a vortex wall, since this minimizes the stray fiel at little cost from the exchange energy term as theoretically predicted. ${ }^{9}$ The micromagnetic simulation in Fig. 2(b) shows remarkable agreement, although the exact position of the head to head domain wall in the experiment is determined by local defects (the highly energetic vortex core is pinned at non-magnetic defects). In addition to the head to head walls, a domain structure is visible in the SEMPA image and in the simulation demonstrating the influenc of the magnetocrystalline anisotropy for this wide ring geometry.

In narrow rings any deviation of the magnetization from parallel to the ring edge will create dipole stray fields Therefore, the magnetization closely follows the shape (local shape anisotropy) and aligns with the circumference. In this case no domain features are expected since the strong local shape anisotropy supersedes the magnetocrystalline anisotropy. This can be seen in the SEMPA image in Fig. 2(c) and in the micromagnetic simulation in Fig. 2(d) of a narrow ring $(D=1600 \mathrm{~nm}, d=1200 \mathrm{~nm}, t=34 \mathrm{~nm}$ fcc Co). Furthermore in this narrow ring a transverse head to head domain wall is present since a vortex wall would have to be compressed into the narrow ring resulting in a prohibitively high exchange
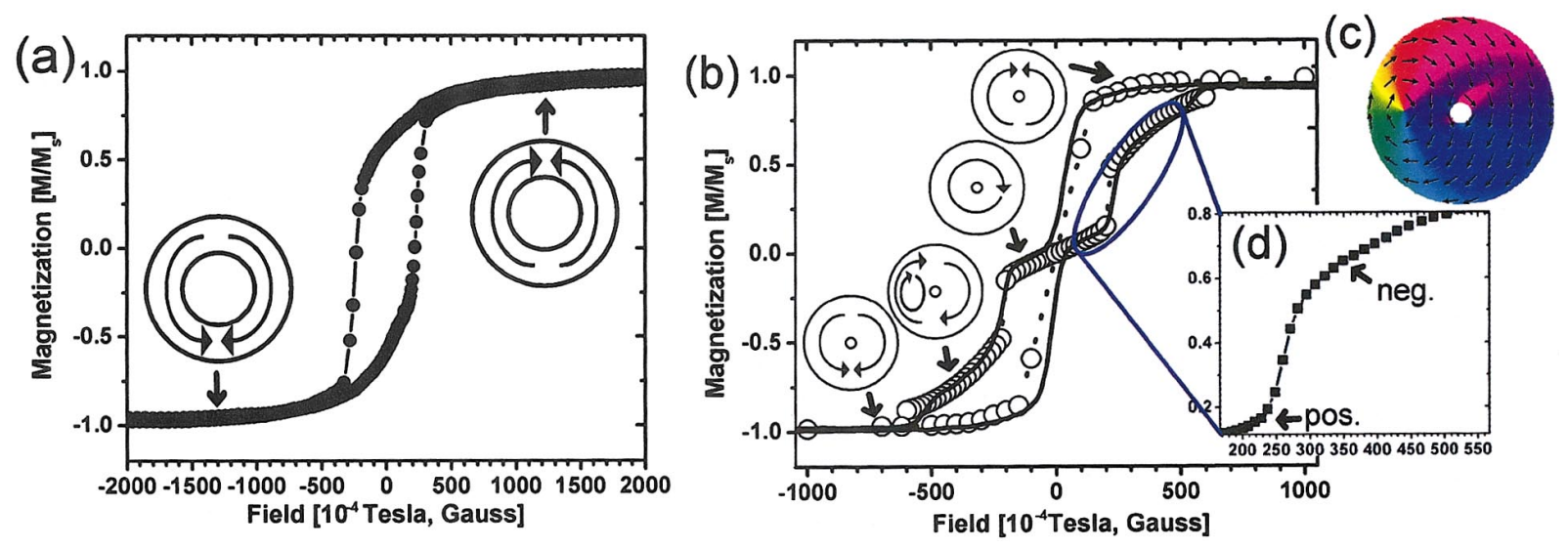

FIG. 3. (Color) MOKE loops of an array of rings. (a) Hysteresis loop showing single switching in thin fil rings ( $D=1700 \mathrm{~nm}, d$ $=1250 \mathrm{~nm}, t=4 \mathrm{~nm}$ polycrystalline Co) with the magnetization configuration of the onion states shown schematically. (b) Triple switching in very wide rings with thick film $(D=1700 \mathrm{~nm}, d=300 \mathrm{~nm}, t=32 \mathrm{~nm}$ polycrystalline Co, experimental data: black line, micromagnetic simulation: empty circles). The magnetization configuration are shown schematically (from top to bottom: onion, vortex, vortexcore and opposite onion state). (c) Micromagnetic simulation of the magnetization configuratio in the vortexcore state (color code as in Fig. 2). (d) Enlargement of the experimental data in the interesting fiel region showing the difference in curvature when the fiel is applied to the ring in the vortex state (indicated with pos.) and in the vortexcore state (indicated with neg.) 
energy. The energetics of the different domain wall types is analyzed further in Ref. 9 and the different kinds of domain walls observed here are in good agreement with Ref. 9.

While the geometry dependence of the remanent magnetic states can be studied with SEMPA, it is difficul to image under an applied magnetic field To study the magnetic states and transitions, which develop when a fiel is applied, we make MOKE measurements on arrays of rings. In the limited range of geometries and materials that have been studied previously ${ }^{3,4,6}$ only the double switching process discussed above was reported. Here we have varied the fil thickness and ring width over a very wide range (fil thickness fcc Co 5-34 nm, polycrystalline Co $2-34 \mathrm{~nm}$, and polycrystalline Permalloy $2-45 \mathrm{~nm}$, ring width $5-90 \%$ of the outer diameter $D, 300 \mathrm{~nm}<D<3000 \mathrm{~nm}$ ). $D$ is kept $<3 \mu \mathrm{m}$ for mesoscopic rings to prevent the occurrence of complicated and defect dominated multidomain states.

In addition to the previously found double switching process $[\text { Fig. 1(a) }]^{3,4}$ depending on the geometry we fin other types of hysteresis behavior: the reversal can occur with a single transition as seen in Fig. 3(a) or with triple switching as seen in Fig. 3(b).

To elucidate these different behaviors we consider the transitions that are available for each magnetic state. After relaxing the fiel from saturation a ring develops the onion state with a wall structure determined by the geometry [see Figs. 2(a)-2(d)]. Additional simulations show that then there are two competing switching processes when a reverse fiel is applied: (i) for rings made of a thick magnetic film domain wall depinning followed by propagation leads to the double switching process as seen in Fig. 1(a) and explained in Refs. 3 and 17; and (ii) in rings with a thin fil a reverse domain can be nucleated somewhere at the edge of the ring (not necessarily related to the position of the domain walls), which then grows and spreads over the whole ring until the opposite onion state is attained. This process is a nucleationpropagation process similar to that described in detail in Ref. 17 but in this case it leads to a single onion to (reverse-) onion switching characterized by a single jump in the $M-H$ loop [Fig. 3(a)]. This process is very unfavorable in thick fil rings since the spins are strongly aligned with the edges. Any deviation from the perimeter would be energetically disadvantageous in this case due to the large dipolar stray fiel created, whereas in thin rings the necessary twisting of spins to nucleate a reverse domain can happen easily. Furthermore in very thin rings the onion state can be energetically favored over the vortex state because of the lower exchange energy, which outweighs the small stray fiel energy of the onion state, so that as seen in Fig. 3(a) a very thin ring $(t=4 \mathrm{~nm}$ here) switches directly from the onion state to the reverse onion state. In the softer permalloy the single switching is observed as well but for thinner rings, since the smaller exchange and saturation magnetization favor the vortex state as compared to cobalt.

To explain the triple switching observed for wide and very thick rings [e.g., the ring geometry for Fig. 3(b)], we have computed a hysteresis loop for such a ring. As seen in Fig. 3(b) the simulation reproduces the experimental loop well and the magnetization configuration found are shown sche- matically. Apart from the onion and vortex state, we observe a new state [schematic in Fig. 3(b) and micromagnetic simulation of the detailed magnetization configuratio in Fig. $3(\mathrm{c})$ ], which we term the "vortexcore" state (since a complete vortex core is present). This state is not stable at remanence, where the ring reverts to the vortex state. This is because both states have a zero stray fiel but the exchange energy is higher in the vortexcore state due to the presence of the vortex core. In an applied field on the other hand, the gain in Zeeman energy in the vortexcore state makes it energetically favorable. As the vortexcore state has to be stabilized by an applied field it could not be directly imaged with the techniques available, but we can compare the simulated and measured hysteresis loops shown in Fig. 3(b). The height of the jumps is well reproduced in the simulation. In addition, a very sensitive measure for the type of magnetization configuratio is the curvature of the loop when the fiel is applied. As predicted earlier ${ }^{18}$ the curvature $d^{2} M / d H^{2}$ of the hysteresis loop is positive when a fiel is applied to a ring in the vortex state and negative if the ring is in a state where a vortex core is present, such as the vortexcore state. As seen in Fig. 3(b) and the enlargement of the interesting fiel region [Fig. 3(d)], the curvature after the firs switching is positive, which means that the rings have switched from the onion state into the vortex state. After the second jump the curvature is negative, which means that the ring has switched from the vortex to the vortexcore state. This negative curvature is the same as that reported for the case of discs, ${ }^{19}$ where by direct imaging of the vortex core ${ }^{20}$ it has been established that the negative curvature is directly correlated to a magnetic state where a vortex core is present. ${ }^{18-20}$ The similarities in the curvature are due to the fact that physically the same process is occurring when a vortex core is present in a disc (vortex state) or in a ring (vortexcore state) when an applied fiel is increased: the component of the magnetization parallel to the fiel direction grows, and hence the vortex core is pushed outwards [in the case of Fig. 3(b) towards the left] until at the critical fiel it leaves the structure and the ring attains the onion state. It is now clear why the vortexcore state is only observed in wide and very thick rings: due to the exchange energy it is only possible to "fit" a vortex core into rings above a certain width and only for large thicknesses the energy gain due to the absence of a stray fiel offsets the higher exchange energy of the vortexcore state.

In conclusion, we have presented the nanoscopic details of the magnetization configuration in mesoscopic rings, which show the influenc of geometry and magnetocrystalline anisotropy particularly on the spin structure of the different types of head to head domain walls. The influenc of the geometry on the switching processes is evident, and three categories of switching behavior are found. In addition to the double switching process, thin rings show a single reversal mechanism, while very wide rings with a thick fil exhibit triple switching where the vortexcore state is shown to occur.

The authors acknowledge support from the Gottlieb Daimler-and Karl Benz Foundation (M.K.) and the Offic of Naval Research (T.L.M. and J.U.). 
*Current address: Fachbereich Physik, Universitaet Konstanz, Universitaetsstr. 10, D-78457 Konstanz, Germany.

${ }^{\dagger}$ Corresponding author. Electronic address: jacb1@phy.cam.ac.uk

${ }^{1}$ L. D. Landau and E. M. Lifshitz, Phys. Z. Sowjetunion 8, 153 (1935).

${ }^{2}$ J.-G. Zhu, Y. Zheng, and G. A. Prinz, J. Appl. Phys. 87, 6668 (2000).

${ }^{3}$ J. Rothman, M. Kläui, L. Lopez-Diaz, C. A. F. Vaz, A. Bleloch, J. A. C. Bland, Z. Cui, and R. Speaks, Phys. Rev. Lett. 86, 1098 (2001).

${ }^{4}$ M. Kläui, J. Rothman, L. Lopez-Diaz, C. A. F. Vaz, J. A. C. Bland, and Z. Cui, Appl. Phys. Lett. 78, 3268 (2001).

${ }^{5}$ M. Kläui, C. A. F. Vaz, J. A. C. Bland, W. Wernsdorfer, G. Faini, and E. Cambril, Appl. Phys. Lett. 81, 108 (2002).

${ }^{6}$ S. P. Li, D. Peyrade, M. Natali, A. Lebib, Y. Chen, U. Ebels, L. D. Buda, and K. Ounadjela, Phys. Rev. Lett. 86, 1102 (2001).

${ }^{7}$ S. Kasai, T. Niiyama, E. Saitoh, and H. Miyajima, J. Magn. Magn. Mater. 239, 228 (2002).

${ }^{8}$ M. Kläui, C. A. F. Vaz, L. Lopez-Diaz, and J. A. C. Bland, J. Phys.: Condens. Matter 15, 985 (2003).

${ }^{9}$ R. McMichael and M. Donahue, IEEE Trans. Magn. 33, 4167 (1997).
${ }^{10}$ M. Kläui, C. A. F. Vaz, J. Rothman, J. A. C. Bland, W. Wernsdorfer, G. Faini, and E. Cambril, Phys. Rev. Lett. 90, 097202 (2003).

${ }^{11}$ L. Lopez-Diaz, M. Kläui, J. Rothman, and J. A. C. Bland, Physica B 306, 211 (2001).

${ }^{12}$ L. Lopez-Diaz, J. Rothman, M. Kläui, and J. A. C. Bland, J. Appl. Phys. 89, 7579 (2001).

${ }^{13}$ C. David and D. Hambach, Microelectron. Eng. 46, 219 (1999).

${ }^{14}$ A. Locatelli, K. S. Cherifi S. Heun, M. Marsi, K. Ono, A. Pavlovska, and E. Bauer, Surf. Rev. Lett. 9, 171 (2002).

${ }^{15}$ M. R. Scheinfein, J. Unguris, M. H. Kelley, D. T. Pierce, and R. J. Celotta, Rev. Sci. Instrum. 61, 2501 (1990).

${ }^{16}$ The free OOMMF package is available at http://math.nist.gov/ oommf.

${ }^{17}$ M. Kläui, L. Lopez-Diaz, J. Rothman, C. A. F. Vaz, J. A. C. Bland, and Z. Cui, J. Magn. Magn. Mater. 240, 7 (2002).

${ }^{18}$ L. Lopez-Diaz, J. Rothman, M. Kläui, and J. A. C. Bland, IEEE Trans. Magn. 36, 3155 (2000).

${ }^{19}$ R. P. Cowburn, D. K. Koltsov, A. O. Adeyeye, M. E. Welland, and D. M. Tricker, Phys. Rev. Lett. 83, 1042 (1999).

${ }^{20}$ T. Shinjo, T. Okuno, R. Hassdorf, K. Shigeto, and T. Ono, Science 289, 930 (2000). 